\title{
Óleo essencial de Melaleuca alternifolia no tratamento da acne
}

\author{
Melaleuca alternifolia essential oil for acne treatment \\ Aceite essencial de Melaleuca alternifolia para el tratamento del acné
}

\section{Resumo}

Objetivo: Entre os diversos óleos essenciais, o óleo essencial de Melaleuca alternifolia se destaca diante das suas múltiplas atividades biológicas, sendo a principal a antimicrobiana, tornando-o um elemento atraente para tratamentos faciais. Dito isso, o objetivo deste estudo é avaliar a eficácia do óleo essencial de Melaleuca alternifolia no tratamento da acne. Métodos: O trabalho desenvolvido caracteriza-se como revisão sistemática da literatura. O levantamento bibliográfico foi realizado nas bases de dados Pubmed, ScienceDirect, Lilacs e Medline. Durante a etapa de busca, se estabeleceram como critérios de inclusão artigos publicados no período de 2011 a 2021, artigos com dados primários e disponíveis em língua inglesa, portuguesa e espanhola. Já os critérios de exclusão foram: artigos que apresentaram incompatibilidade com o tema, duplicidade nas bases e artigos de revisão. Resultados: Após a realização das buscas nas bases de dados, da aplicação dos critérios de inclusão e leitura do título e resumo, foram selecionados dez artigos que se enquadravam nos requisitos de elegibilidade. Os dez artigos foram lidos na íntegra, com análise acurada de suas metodologias e principais resultados. Considerações finais: $\mathrm{O}$ óleo de melaleuca apresenta um grande potencial no tratamento da acne devido sua significativa atividade antimicrobiana, capacidade de reduzir lesões, bem como auxiliar na cicatrização de feridas. Ademais, apresenta vantagens em relação ao uso de antibióticos devido ao baixo impacto no desenvolvimento de resistência e suscetibilidade aos antimicrobianos. Ainda que apresente estudos acerca das atividades farmacológicas deste óleo, é imprescindível a realização de pesquisas mais apuradas para consolidar esses apanhados.

Palavras-chave: Melaleuca alternifolia; Acne; Óleo essencial.

\begin{abstract}
Objective: Among the various essential oils, the essential oil of Melaleuca alternifolia stands out for its multiple biological activities, the main one being antimicrobial, which makes it an important element for facial treatments. That said, the goal of this study is to evaluate the effectiveness of Melaleuca alternifolia essential oil in treating acne. Methods: the work carried out is characterized as a systematic literature review. The bibliographic survey was carried out in the Pubmed, ScienceDirect, Lilacs and Medline databases. During the search stage, articles published in the period from 2011 to 2021, articles with primary data and available in English, Portuguese and Spanish were established as inclusion criteria. On the other hand, the exclusion criteria were: articles that presented incompatibility with the topic, duplication in the bases and review articles. Results: after searching the databases, applying the inclusion criteria and reading the title and abstract, ten articles were selected that met the eligibility requirements. The ten articles were read in full, with a refined analysis of their methodology and the main results. Final considerations: melaleuca oil has great potential in the treatment of acne due to its important antimicrobial activity, ability to reduce injuries, as well as helping wound healing. In addition, it has advantages in relation to the use of antibiotics due to the low impact on the development of resistance and susceptibility to antimicrobials. Despite there are studies about this oil's pharmacological activities, more researches are essential to consolidate it.
\end{abstract}

Keywords: Melaleuca alternifolia; Acne; Essential oil. 


\begin{abstract}
Resumen
Objetivo: Entre los diversos aceites esenciales, el aceite esencial de Melaleuca alternifolia se destaca por sus múltiples actividades biológicas, siendo la principal la antimicrobiana, lo que la convierte en un elemento importante para tratamientos faciales. Dicho esto, el objetivo de este estúdio es evaluar la eficacia del aceite esencial de Melaleuca alternifolia en el tratamiento del acné. Métodos: el trabajo desarrollado se caracteriza como una revisión sistemática de literatura. El levantamiento bibliográfico fue realizado en las bases de datos Pubmed, ScienceDirect, Lilacs y Medline. Durante la etapa de búsqueda, se establecieron como criterios de inclusión artículos publicados en el periodo de 2011 a 2021, artículos con datos primarios y disponibles en el idioma inglés, portugués y español. Por su parte, los criterios de exclusión fueron: artículos que presentaron incompatibilidad con el tema, duplicidad en las bases y artículos de revisión. Resultados: después de realizar la búsqueda en las bases de datos, de la aplicación de los criterios de inclusión y la lectura del título y el resumen, fueron seleccionados diez artículos que encajaban con los requisitos de elegibilidad. Los diez artículos fueron leídos por completo, con un análisis depurado de su metodología y los principales resultados. Consideraciones finales: el aceite de melaleuca presenta un gran potencial en el tratamiento del acné debido a su importante actividad antimicrobiana, capacidad de reducir lesiones, así como ayudando en la cicatrización de heridas. Además de eso, presenta ventajas en relación al uso de antibióticos debido al bajo impacto en el desarrollo de resistencia y susceptibilidad a los antimicrobianos. Aún que presenta estudios sobre las actividades farmacológicas de este aceite, es fundamental realizar investigaciones más detalladas para consolidar estos hallazgos.
\end{abstract}

Palabras clave: Melaleuca alternifolia; Acné; Aceite esencial.

\title{
1. Introdução
}

Os óleos essenciais também conhecidos como óleos voláteis, derivam de metabólitos secundários das plantas aromáticas. São substâncias complexas e de fragrância variável, proveniente de qualquer parte da planta (Brito et al., 2013). Estes compostos são constituídos por álcoois, aldeídos, cetonas, fenóis, ésteres e apresentam em sua composição, principalmente, monoterpenos, sesquiterpenos e fenilpropanoides, que são responsáveis por modular suas propriedades terapêuticas (Bruno \& Almeida, 2021; Khodaei et al., 2021).

As ações terapêuticas dos óleos essenciais são diversificadas e muito amplas. Por muitos anos, esses óleos têm sido usados para tratar doenças. As atividades farmacológicas principais são: ação antibacteriana, anti-inflamatória, antioxidantes, antiviral, dentre outras. Além disso, são substâncias que possuem matérias-primas importantes para as indústrias medicinal, cosmética, farmacêutica e alimentícia (Bruno \& Almeida, 2021; Pazyar et al., 2013; Paviani et al., 2019; Zhu et al., 2020).

Entre os diversos tipos de óleos essenciais, destaca-se o óleo essencial de melaleuca, também conhecido como Tea Tree Oil (TTO), o qual é de espécie arbórea nativa da Austrália, pertencente à família Myrtaceae (Hammer, 2015). A Melaleuca alternifolia é um arbusto alto de até $15 \mathrm{~m}$ de altura com uma copa espessa e de casca fina, com odor característico de terpeno, conífero e menta-cânfora (de Groot \& Schmidt, 2016).

Outrossim, o óleo essencial de melaleuca contém mais de 100 componentes voláteis, os quais são principalmente hidrocarbonetos oxigenados, monoterpenos monocíclicos e monoterpenos bicíclicos (Lam et al., 2020). O $\beta$-pineno, $\alpha$ terpineol, $\rho$-cimeno, terpinen-4 ol, 1,8-cineol, y-terpineno, $\alpha$-terpineno são os seus principais constituintes (De Groot \& Schmidt, 2016), sendo o terminen-4-ol o mais abundante, compreendendo-se $40 \%$ do óleo. As múltiplas atividades biológicas da melaleuca tornam o óleo um elemento atraente para tratamentos faciais, destacando-se o tratamento da acne. Dentre elas, destacam-se as atividades bactericida, antiviral, antifúngica, anti-inflamatória, antitumoral, analgésica, inseticida e acaricida (de Groot \& Schmidt, 2016; Hammer, 2015).

A acne é uma doença de pele inflamatória crônica que afeta principalmente adolescentes e jovens adultos e é causada por diversos fatores que estão associados a uma produção excessiva de sebo, descamação anormal do epitélio folicular, inflamação e aumento da colonização por Propionibacterium acnes, conhecida atualmente como Cutibacterium acnes (Hammer, 2015). As principais características clínicas da acne são: seborreia (excesso de gordura), lesões não inflamatórias (comedões abertos e fechados), lesões inflamatórias (pápulas e pústulas) e diferentes graus de cicatrizes (Williams et al., 2012). Embora na maioria dos pacientes a acne possa ser limitada a uma pequena quantidade de pápulas ou comedões, em 
casos de uma doença grave pode causar cicatrizes desfigurantes no rosto levando a consequências psicossociais graves, causando baixa autoestima, isolamento social e depressão (Mazzarello et al., 2011).

O tratamento da acne engloba tratamentos tópicos com retinóides ou antibióticos, e tratamentos sistêmicos com o uso de antibióticos orais que, podem vir a criar resistência aos microrganismos, além de demostrarem diversos efeitos colaterais como irritação cutânea, gastrointestinal, nefrotoxicidade e fotossensibilidade. Por isso, recentes estudos têm focado em busca de produtos de origem natural que sejam menos irritantes e eficazes frente a essa condição (Ossa-Tabares et al., 2020).

A atividade antimicrobiana do óleo essencial de Melaleuca alternifolia deve-se ao seu mecanismo farmacológico que compromete a integridade da membrana celular, com consequente perda de material intracelular, gerando assim a incapacidade de manter a homeostase e inibição da respiração (Oliveira et al., 2011). A presença do terpinen-4-ol na composição, o constituinte mais abundante deste óleo, é o principal responsável por exibir as propriedades antimicrobianas e antiinflamatórias (Brun et al., 2019; Pazyar et al., 2013). Diante deste contexto, este estudo teve como objetivo avaliar a eficácia do óleo essencial de Melaleuca alternifolia no tratamento da acne.

\section{Metodologia}

$\mathrm{O}$ trabalho desenvolvido caracteriza-se como revisão sistemática da literatura baseada na pergunta norteadora "O óleo essencial de Melaleuca alternifolia é eficaz no tratamento da acne?”. Este tipo de revisão se caracteriza por sintetizar resultados de estudos primários nos quais se enquadram em critérios de elegibilidade com objetivo de responder uma pergunta de pesquisa. A busca por dados é realizada de forma sistemática, por meio de várias fontes de dados, utilizando uma estratégia de busca ampla e sensível. Ademais, esse tipo de estudo evita desperdícios de pesquisa, garantindo assim, que novas pesquisas primárias possam ser realizadas com pleno conhecimento da evidência existente (Ministério da Saúde, 2021).

O estudo foi desenvolvido com base em critérios de pesquisa, identificação, seleção, elegibilidade e inclusão dos estudos identificados nas bases de dados utilizadas. As informações contidas nesse estudo designam-se de artigos científicos sobre a temática, sendo acessados nas seguintes bases de dados: Pubmed, ScienceDirect, Literatura Latino-Americana e do Caribe em Ciências da Saúde (LILACS) e Medical Literature Analysis and Retrieval System Online (MEDLINE), sendo as duas últimas acessadas através da Biblioteca Virtual em Saúde (BVS).

Durante a etapa de busca, estabeleceram-se os seguintes critérios de inclusão: artigos publicados no período de 2011 a 2021 (a escolha de tal período se deu por entender que os últimos 10 anos retratam pesquisas mais recentes sobre esta temática); artigos com dados primários e disponíveis em língua inglesa, portuguesa e espanhola. Já os critérios de exclusão foram: artigos que apresentaram incompatibilidade com o tema; duplicidade nas bases e artigos de revisão.

Para a realização da busca nas bases de dados, foram escolhidos os seguintes termos: "Melaleuca alternifolia", "acne" e "óleo essencial" em língua portuguesa e "essential oil" em língua inglesa.

Os descritores escritos em português foram usados na base Lilacs e Medline utilizando o operador booleano OR e AND. Na Lilacs utilizou-se da seguinte forma: "Melaleuca alternifolia" OR "óleo essencial" OR "Acne" e "Melaleuca alternifolia" AND "óleo essencial"; na Medline: "Melaleuca alternifolia" OR "óleo essencial" OR "acne" e em inglês "Melaleuca alternifolia" AND "essential oil". Nas bases de dados PubMed e SienceDirect os termos foram escritos exclusivamente em inglês, sendo a busca realizada par a par com o operador booleano AND. Na PubMed utilizou-se os termos "Melaleuca alternifolia" AND "essential oil" e no ScienceDirect "Melaleuca alternifolia" AND "acne". 


\section{Resultados}

Após a realização das buscas nas bases de dados supracitadas, da aplicação dos critérios de inclusão e leitura do título e resumo, foram selecionados 10 artigos que se enquadravam nos requisitos de elegibilidade, conforme demonstrado no fluxograma (Figura 1).

Figura 1. Fluxograma indicativo do processo de seleção dos estudos selecionados.

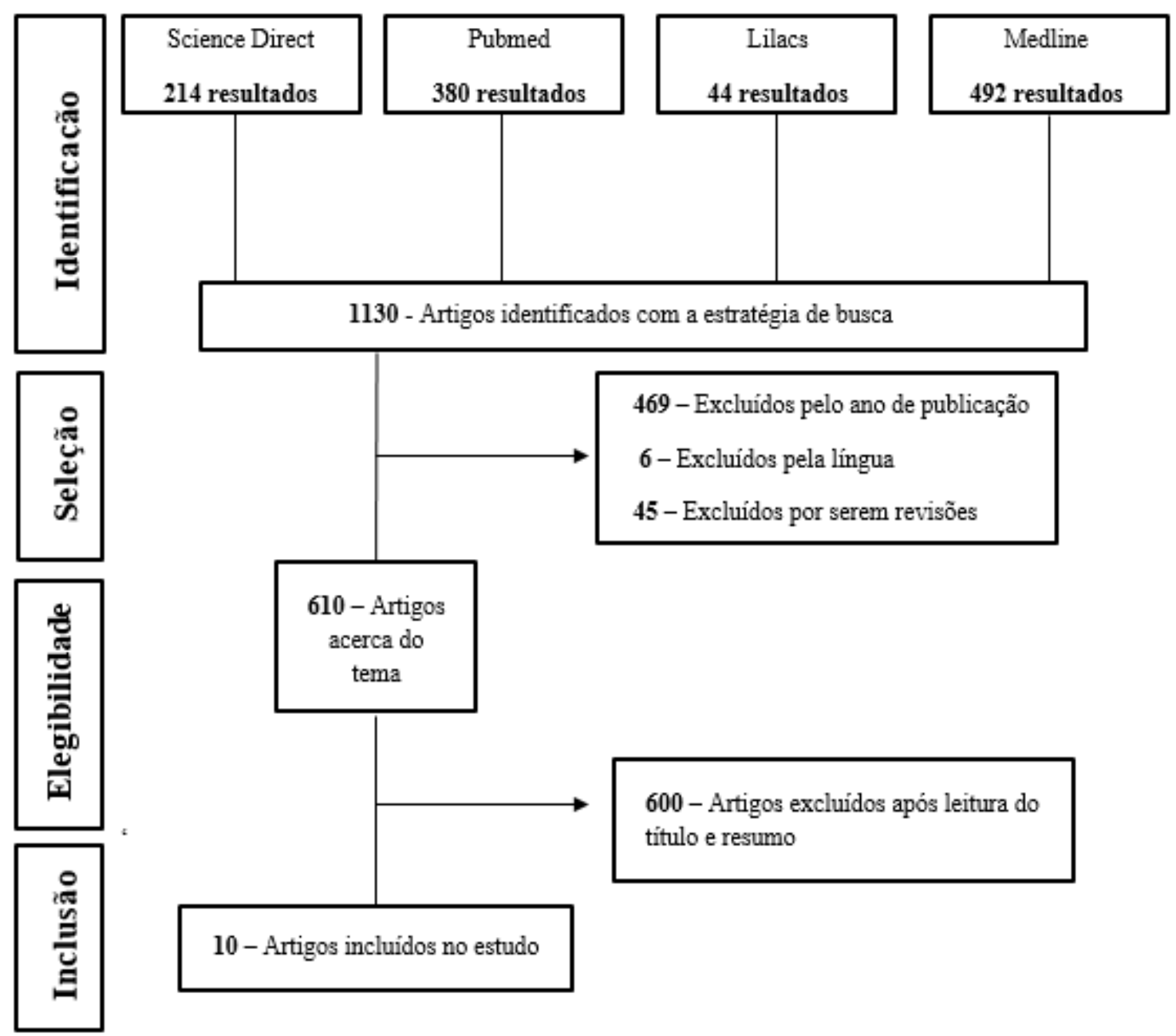

Fonte: Autores (2021).

Os dez artigos incluídos após aplicação dos critérios de inclusão e exclusão, foram lidos na íntegra, com análise acurada de suas metodologias e principais resultados, os quais estão alistados na Tabela 1. 
Tabela 1: Síntese dos estudos selecionados com base no título do artigo, autor(es), ano de publicação, objetivo, tipo de estudo e síntese dos resultados.

\begin{tabular}{|c|c|c|c|}
\hline Título do estudo & Autor/Ano & Objetivo & Periódico \\
\hline $\begin{array}{l}\text { Antimicrobial activity of } \\
\text { certain natural-based plant } \\
\text { oils against the antibiotic- } \\
\text { resistant acne bacteria }\end{array}$ & Esmael et al. (2019) & $\begin{array}{l}\text { Investigar a atividade antibacteriana de } \\
\text { alguns óleos essenciais contra bactérias } \\
\text { indutoras de acne (Staphylococcus } \\
\text { aureus, Staphylococcus epidermidis e } \\
\text { Cutibacterium acnes). }\end{array}$ & $\begin{array}{l}\text { Saudi Journal of Biological } \\
\text { Sciences }\end{array}$ \\
\hline $\begin{array}{l}\text { Correlations of the } \\
\text { componentes of tea tree oil } \\
\text { with its antibacterial effects } \\
\text { and skin irritation }\end{array}$ & Lee et al. (2013) & $\begin{array}{l}\text { Avaliar as correlações dos componentes } \\
\text { do óleo essencial de melaleuca com } \\
\text { suas atividades antiacne e irritação } \\
\text { cutânea. }\end{array}$ & Journal of Food and Drug Analysis \\
\hline $\begin{array}{l}\text { Tea tree oil gel for mild to } \\
\text { moderate acne; a } 12 \text { week } \\
\text { uncontrolled, open-label } \\
\text { phase ii pilot study }\end{array}$ & Malhi et al. (2017) & $\begin{array}{l}\text { Avaliar a eficácia, tolerabilidade e } \\
\text { aceitabilidade de um gel de óleo de } \\
\text { melaleuca disponível comercialmente } \\
\text { para o tratamento da acne leve a } \\
\text { moderada. }\end{array}$ & $\begin{array}{c}\text { Australasian Journal of } \\
\text { Dermatology }\end{array}$ \\
\hline $\begin{array}{l}\text { Evaluación de las } \\
\text { características fisicoquímicas } \\
\text { y de la actividad } \\
\text { antimicrobiana del aceite del } \\
\text { árbol de té contra } \\
\text { Cutibacterium } \\
\text { (Propionibacterium acnes } \\
\text { ATCC } 6919\end{array}$ & $\begin{array}{l}\text { Ossa-Tabares et al. } \\
\qquad(2020)\end{array}$ & $\begin{array}{l}\text { Avaliar a composição, as características } \\
\text { e o efeito antimicrobiano do óleo } \\
\text { essencial de melaleuca a } 2 \% \text { e sua } \\
\text { Concentração Inibitoria Mínima (CIM) } \\
\text { contra Cutibacterium acnes } \\
\text { (Propionibacterium acnes). }\end{array}$ & $\begin{array}{c}\text { Revista Biomédica del Instituto } \\
\text { Nacional de Salud }\end{array}$ \\
\hline
\end{tabular}

Effects of Melaleuca

alternifolia (tea tree) essential

oil and the major

monoterpene component

terpinen-4-ol on the

development of single- and

multistep antibiotic resistance

and antimicrobial

susceptibility

Effect of tea tree (Melaleuca alternifólia) oil as a natural antimicrobial agente in lipophilic formulations
Examinar se o óleo de melaleuca ou seu componente principal, terpinen-4-ol, desenvolve uma nova resistência a antibióticos em bactérias Staphylococcus aureus e Escherichia coli.
American Society for Microbiology (ASM)

\begin{tabular}{|c|c|}
\hline Vitro Antimicrobial & \\
\hline $\begin{array}{lr}\text { Activities of } & \text { Commercially } \\
\text { Available } & \text { Tea Tree } \\
\text { (Melaleuca } & \text { alternifolia) } \\
\text { Essential Oils } & \end{array}$ & Brun et al. (2018) \\
\hline $\begin{array}{l}\text { Avaliação antibacteriana do } \\
\text { extrato de melaleuca } \\
\text { (Melaleuca } \\
\text { frente alternifolia) } \\
\text { Staphylococcus aureus }\end{array}$ & Tedesco et al. (2014) \\
\hline
\end{tabular}

Zhang et al. (2018)

Comparar a atividade antimicrobiana in vitro do TTO e seus componentes principais com os conservantes parabenos tradicionais frente a determinados microrganismos.
Investigar a atividade antimicrobiana e o perfil químico de dez óleos essenciais de Melaleuca disponíveis comercialmente.
Comparar e comprovar o efeito antimicrobiano do óleo essencial comercial de Melaleuca alternifolia e do óleo extraído direto da planta frente a cepa de Staphylococcus aureus. Avaliar as atividades antioxidantes e antimicrobianas do óleo essencial de Melaleuca alternifolia através de amostras coletadas em quatro locais da

\section{Canadian Journal of Microbiology}

\section{In Vitro Evaluation of}

Antioxidant and

Antimicrobial Activities of

Melaleuca alternifolia

Essential Oil cidade de Zhaoqing, província de Guangdong, por meio do método cromatógrafo de gás.

The effect of tea tree oil (Melaleuca alternifólia) on wound healing using a dressing model
Determinar os efeitos antimicrobianos Chin e Cordell (2013) dos vapores do óleo essencial de melaleuca.

\section{Current Microbiology}

Arq. ciências saúde UNIPAR

BioMed Research International

The Journal of Alternative and

Complementary Medicine

Fonte: Autores (2021). 


\section{Discussão}

Acne vulgaris é uma doença de pele que prevalece em todo o mundo. A Propionibacterium acnes, a Staphylococcus epidermidis e a Staphylococcus aureus são as bactérias associadas à patogenicidade dessa doença. Consequentemente, no que se refere ao tratamento, a maioria dos medicamentos antiacne são direcionados contra estas bactérias, infecções e respostas inflamatórias associadas (Taleb et al., 2018).

A terapia antiacne inclui tratamentos tópicos e sistêmicos com anti-inflamatórios, retinóides e antibióticos. Todavia, a resistência bacteriana acompanha os antibióticos e é atribuída a vários fatores, como: concentrações subinibitórias ou uso por tempo prolongado. Além disso, esta resistência, bem como o uso recorrente dos antimicrobianos pode vir a acarretar diversos efeitos colaterais, incluindo irritação cutânea e gastrointestinal, fotossensibilidade e neurotoxicidade. Para superar a resistência aos antibióticos convencionais, agentes antimicrobianos naturais, como os óleos essenciais, vem se tornando um potencial substituto de tratamento para acne (Taleb et al., 2018; Zaenglein, 2018). Dentre os diversos óleos, o de Melaleuca alternifolia é um dos destaques, principalmente por sua atividade antimicrobiana (Winkelman, 2018).

Por meio do estudo realizado por Esmael et al. (2019), foi descrito o isolamento das bactérias Staphylococcus aureus, Staphylococcus epidermidis e Cutibacterium acnes, as quais são responsáveis pelo desenvolvimento da acne, no intuito de avaliar, por meio do método de difusão em ágar, os efeitos antimicrobianos de onze óleos essenciais frente a estas bactérias. Foram selecionados cinquenta e cinco pacientes (20 homens e 35 mulheres), na faixa etária de 17 a 25 anos por meio do exame de diagnóstico patológico, os quais continham lesões cutâneas de acne inflamatória potencial. Porém, apenas 35 dos 55 pacientes apresentaram crescimento bacteriano após o cultivo aeróbico e anaeróbico das amostras coletadas.

Dentre os óleos essenciais avaliados, o de melaleuca e o de alecrim se destacaram na atividade antimicrobiana. Todavia, o óleo essencial de melaleuca mostrou ser mais eficaz contra as bactérias indutoras da acne, tornando-o uma boa opção para substituir os antibióticos no tratamento desta patologia (Esmael et al., 2019).

O óleo essencial de melaleuca vem a ser constituído por mais de 100 componentes e possui como constituintes principais: hidrocarbonetos terpênicos, como monoterpenos, sesquiterpenos e seus álcoois associados (Yadav et al., 2016). As atividades inibitórias dos componentes do TTO e TTO- L (TTO isolados das folhas) contra Propionibacterium acnes e Staphylococcus aureus foram avaliadas, por meio do estudo de Lee et al. (2013), e constatou-se que, os componentes terpinen4-ol, $\alpha$-terpineno, 1,8-cineol, $\rho$-cymene, $\alpha$-pinene, $\alpha$-terpineol e limoneno exibiram atividades antimicrobianas notáveis contra $P$. acnes, dando-se destaque ao terpinen-4-ol e $\alpha$-terpineol. Já no que se refere a Staphylococcus aureus, os componentes que se identificaram como os mais eficazes foram o $\alpha$-terpinoleno e o $\alpha$-terpineno. Dessa maneira, pode ser observado que as atividades antimicrobianas do TTO são atribuídas principalmente ao terpinen-4-ol, $\alpha$-terpineol, $\alpha$-terpinoleno e $\alpha$-terpineno.

Em relação à toxicidade e efeitos do TTO-L, o mesmo estudo realizou um ensaio de irritação da pele e avaliação da função hepática em ratos Wistar utilizando o TTO-L e dois componentes principais: terpinen-4-ol e 1,8-cineol, o principal componente tóxico. Na concentração de $10 \%$, no período de 24 horas, encontraram-se eritemas bem definidos e edemas muito leves e não houve alterações mesmo com o aumento no tempo de aplicação (48 horas). Todavia, a irritação diminuiu ao reduzir a concentração em 5\%. Já em 2,5\% nenhuma irritação na pele foi observada. Isto é, mesmo com o aumento do tempo de aplicação e a porcentagem dos componentes, os níveis de eritema e edema se mostraram baixos e leves. Dessa forma, evidenciou-se que o óleo essencial de Melaleuca alternifolia apresenta baixa toxicidade e boa segurança (Lee et al., 2013).

Isso também pode ser observado no estudo piloto de fase II de centro duplo, realizado na Austrália Ocidental, com participantes de ambos os sexos, de faixa etária entre 16 e 39 anos, com acne facial leve a moderada (Malhi et al., 2017), os quais receberam produtos com o gel de óleo de melaleuca sendo instruídos a usá-los duas vezes ao dia, e mantê-los na pele por pelo menos seis horas. Após a avaliação clínica da eficácia e tolerabilidade, a maioria dos participantes ficaram satisfeitos na redução significativa do número das lesões durante o tratamento. Ademais, o tratamento foi bem tolerado, ou seja, não 
ocorreram eventos adversos graves, apenas descamação e secura moderada e leve coceira nos primeiros dias após a aplicação dos produtos. Ato contínuo, a aceitabilidade dos produtos foi alta. As pontuações mais baixas para aceitação foram devido à fragrância e absorção, enquanto as mais altas foram para textura, consistência e facilidade de aplicação.

Para avaliar a atividade antibacteriana de três lotes distintos de óleo essencial de melaleuca, Ossa-Tabares et al. (2020), utilizaram o ensaio de difusão em ágar. Os autores observaram o efeito inibitório sobre o crescimento do microrganismo em todas as placas dos diferentes lotes de óleo na concentração de $2 \%$ v/v contra a cepa de Cutibacterium acnes, uma vez que o valor da Concentração Inibitória Mínima (CIM) aproximou-se ao menor valor das faixas relatadas na literatura. Esta atividade antibacteriana do óleo de melaleuca, diante de alguns estudos, deve-se à perda de integridade da membrana e da inibição da respiração após sua aplicação (Yadav et al., 2016).

No mesmo estudo avaliou-se e quantificou-se a composição, por meio da cromatografia gasosa, e as características físico-químicas (cor, densidade e índice de refração) do óleo essencial de melaleuca. O terpinen-4-ol foi o componente mais abundante, com concentração entre 40,7 e 43,9\%, seguido por $\gamma$-terpineno, com valores entre 20,7 e 22,6\%, e $\alpha$-terpineno, com uma concentração entre 9,5 e 10,5\%. Os demais componentes atenderam à faixa especificada na norma ISO 4730. Por outro lado, as características físico-químicas descritas atenderam ao disposto desta norma, indicando uma alta qualidade do óleo essencial de melaleuca (Ossa-Tabares et al., 2020).

Hammer et al. (2012), baseou-se no óleo essencial de melaleuca e no seu principal componente, terpinen-4-ol, frente ao desenvolvimento da resistência a antibióticos (ciprofloxacino, vancomicina, mupirocina, rifamicina, canamicina e ampicilina), das bactérias Staphylococcus aureus e Escherichia coli. As frequências de mutantes resistentes a estes antibióticos de etapa única foram estabelecidas inoculando bactérias, cultivadas com e sem óleo de melaleuca, em ágar contendo 2 a 8 vezes a CIM de cada antibiótico.

Embora os experimentos acima apontaram poucas diferenças nas frequências de resistência, a combinação do antibiótico canamicina com o óleo essencial de melaleuca desenvolveu cerca de 10 vezes menos mutantes de Escherichia coli resistentes em comparação à canamicina isolada. Todavia, nos resultados, em geral, o óleo de melaleuca juntamente com o terpinen-4-ol demonstraram pouco impacto no desenvolvimento de resistência e suscetibilidade aos antimicrobianos. Ou seja, há poucos indícios para preocupar-se na elevação da resistência antimicrobiana com o uso frequente deste óleo (Hammer et al., 2012). Desse modo, o uso do óleo essencial de melaleuca torna-se uma boa opção quando comparado aos antimicrobianos, uma vez que, estes medicamentos podem desencadear cepas de P.acnes com resistência a diferentes antibióticos, resultando tanto em possíveis implicações na acne, quanto em outras doenças que a bactéria possa atuar como patógeno (Dessinioti \& Katsambas, 2017).

Mantil et al. (2015) realizaram um estudo comparativo entre atividade antimicrobiana in vitro do óleo essencial de melaleuca e seus principais componentes com os conservantes parabenos tradicionais frente aos microrganismos Saccharomyces cerevisiae, Pythium sulcatum, Escherichia coli, Bacillus subtilis, Rhizopus stolonifer e Botrytis cinerea. Ainda por cima, determinou-se a atividade das propriedades antimicrobianas do óleo como um agente antimicrobiano em um produto lipofílico.

A determinação do efeito do óleo sobre os microrganismos testados, resultaram na sua incorporação aos meios de cultura Potato Dextrose Agar (PDA) e ao Agar Triptona de Soja (TSA) a fim de obter as concentrações 0,008\%, 0,08\% e 0,8\% $(\mathrm{m} / \mathrm{m})$. Os resultados apresentados mostraram que na concentração de $0,8 \%$ o óleo essencial de melaleuca demonstrou um efeito significativo na redução do crescimento de todos os microrganismos (Mantil et al., 2015).

Nas bactérias Escherichia.coli e Bacilus subtilis, esta concentração demonstrou ser tão eficaz quanto aos parabenos. Ainda mais, o fungo Rhizopus stolonifer, apresentou $100 \%$ de inibição de crescimento com o TTO e metilparabeo, já com o propilparabeno, a redução foi de 51\%. A levedura Saccharomyces cerevisiae teve seu crescimento reduzido (80\%-100\%) no 
tratamento com parabenos, bem como nas concentrações de 0,8\% e o 0,08\% do óleo. Os fungos Botrytis cinerea e Pythium sulcatum, demonstraram maior redução no crescimento na concentração de $0,8 \%$ (de $93 \%-100 \%$ para Botrytis cinerea e $200 \%$ para Pythium sulcatum). Ainda, a 0,08\%, o óleo também inibiu de forma significativa o crescimento de Botrytis cinerea e Pythium sulcatum em $56 \%$ e $78 \%$, respectivamente. Em suma, as concentrações mais altas de TTO $(0,08 \%$ e $0,8 \%)$ foram consideradas tão eficazes quanto os parabenos (metil- e (ou) propilparabeno) na redução do crescimento microbiano (Mantil et al., 2015).

Em relação aos principais componentes do óleo de melaleuca e suas atividades antimicrobianas, o terpinen-4-ol e o yterpineno apresentaram-se como os mais eficazes na inibição do crescimento microbiano. Todavia, os componentes individuais do óleo não obtiveram um efeito tão significante quando comparado ao TTO inteiro. Em outras palavras, os componentes podem atuar de forma sinérgica para fornecer o efeito total do óleo essencial de Melaleuca alternifólia (Mantil et al., 2015).

Os parabenos são compostos muito utilizados na indústria cosmética há anos. Sua solubilidade adequada permite sua atividade como conservante antimicrobiano (Ferreira, 2019). Porém, entre inúmeras substâncias que são capazes de causar reações indesejadas, os conservantes, como os parabenos, de maneira geral, relataram possuir sensibilizantes causadores de alergia e dermatites de contato em formulações de produtos para a pele. Outra característica que possibilita a ocorrência dos efeitos indesejáveis dos parabenos é a sua alta lipofilicidade e seu baixo peso molecular que faz com que a sua absorção venha a ocorrer com mais facilidade (Hoppe, 2017).

Os autores Brun et al. (2018) também não foram capazes de atribuir a atividade antimicrobiana do componente terpinen-4-ol devido a variabilidade na composição do óleo de melaleuca, concluindo que esta atividade é resultado das interações complexas entre os diversos constituintes. Esta conclusão deu-se por meio de experimentos in vitro realizados com dez lotes (T1-T10) de óleo essencial de melaleuca, adquiridos no mercado italiano, os quais foram investigados e avaliados frente a atividade antimicrobiana contra fungos resistentes à meticilina Staphylococcus aureus (MRSA), e Pseudomonas aeruginosa cultivado por meio de biofilmes. Dos dez lotes, apenas cinco apresentaram atividades antimicrobianas significativas, uma vez que, reduziram a sobrevivência das bactérias nos biofilmes, resultando em danos oxidativos nos fungos.

Em outro estudo realizado por Tedesco et al. (2014), através da técnica Pour Plate, comparou-se e comprovou-se o efeito antimicrobiano de dois óleos essenciais de melaleuca disponíveis comercialmente (definidos no estudo como A e B) e do óleo extraído direto da planta nas diferentes épocas sazonais, frente a cepa de Staphylococcus aureus. No mês de junho, o óleo essencial extraído diretamente da planta obteve melhor resultado de inibição microbiana quando comparado com os outros óleos comerciais (A e B). Estes resultados demonstram que o óleo essencial de Melaleuca alternifolia apresenta uma importância significativa frente ao efeito bactericida em Staphylococcus aureus.

A atividade antibacteriana do óleo essencial de M. alternifolia também foi investigada por Zhang et al. (2018), com três métodos diferentes, sendo eles: o método de triagem antimicrobiana e os ensaios CIM e CBM (Concentração Bactericida Mínima). Os resultados demostraram que este óleo exibe uma grande e significativa atividade antimicrobiana contra todos os microrganismos que foram testados, dentre eles: Escherichia coli, Staphylococcus aureus, Pseudomonas aeruginosa, Penicillium italicum Wehmer e Penicillium digitatum Sacc. Ainda, foi-se observado que as bactérias gram-positivas eram mais sensíveis ao óleo essencial do que as bactérias gram-negativas.

Além de avaliar as propriedades antibacterianas, o estudo também investigou o potencial antioxidante do óleo essencial de $M$. alternifolia em comparação com antioxidantes naturais. Os resultados exibiram fortes propriedades na eliminação de radicais livres e inibição da peroxidação lipídica, evidenciando um ótimo potencial antioxidativo. Ademais, os estudos comprovaram que, os hidrocarbonetos monoterpênicos são os melhores compostos antioxidantes em relação aos sesquiterpenos, destacando-se, o terpineno-4-ol, y-terpineno e $\alpha$-terpineno (Zhang et al., 2018). 
Essas propriedades provavelmente se devem à atividade inerente que alguns componentes presentes no óleo essencial possuem, em particular os fenóis, que inibem ou reduzem a taxa de oxidação aeróbica da matéria orgânica (Amorati et al., 2013).

Ainda por cima, já há evidências dos óleos essenciais frente à cicatrização de feridas, bem como sua possível aplicação na prática clínica (Payzar et al., 2013). Isso por ser observado no estudo realizado por Chin e Cordell (2013), o qual comparou o tempo de cicatrização de feridas com abcessos infectados por $S$. aureus entre o tratamento com antibiótico e o tratamento convencional com óleo essencial de melaleuca. Dessa forma, observou-se que este óleo pode ajudar na cicatrização de forma acelerada, além de ser utilizado como um tipo de terapia complementar mais segura no tratamento de infecções leves e comuns. Todavia, os pacientes que realizaram o tratamento de melaleuca juntamente com os antibióticos apresentaram uma resposta curativa ainda mais rápida.

O óleo essencial de Melaleuca alternifolia é um produto que pode ser utilizado como terapia complementar e substitutiva, que apresenta diversas atividades biológicas e acentua-se por exibir uma grande e significativa atividade antimicrobiana frente a diversos microrganismos, principalmente contra as bactérias indutoras da acne. Este óleo apresenta um potencial significativo para o seu uso isolado ou incorporado em cosméticos e produtos farmacêuticos, devido a sua capacidade de reduzir lesões, bem como auxiliar na cicatrização de feridas oriundas desta patologia.

É inegável que este óleo possui uma boa tolerabilidade e segurança por apresentar poucos efeitos adversos e demonstrar pouco impacto no desenvolvimento de resistência e suscetibilidade aos antimicrobianos, uma vez que, estes medicamentos podem desencadear cepas de bactérias resistentes. Todavia, é importante salientar que o óleo de melaleuca pode tornar-se tóxico à medida que não seja utilizado de forma segura, isto é, quando aplicado excessivamente, diretamente sem diluição e com concentração inadequada.

\section{Considerações Finais}

Em suma, é possível concluir que, embora haja muitos estudos clínicos a respeito das atividades farmacológicas do óleo de melaleuca, faz-se necessário mais pesquisas refinadas para corroborar esses achados, bem como para auxiliar sua inserção nas formulações de produtos farmacêuticos. Outrossim, esta revisão pode fornecer uma infinidade de informações úteis para pesquisadores que desejam realizar novos estudos nesta área de conhecimento.

\section{Referências}

Amorati, R., Foti, M. C., \& Valgimigli, L. (2013). Antioxidant activity of essential oils. Journal of agricultural and food chemistry, 61(46), 10835-10847. 10.1021/jf403496k

Brito, A. M. G., Rodrigues, S. A., Brito, R. G., \& Xavier-Filho, L. (2013). Aromaterapia: da gênese a atualidade. Revista Brasileira de plantas medicinais, 15, 789-793. https://doi.org/10.1590/S1516-05722013000500021

Brun, P., Bernabè, G., Filippini, R., \& Piovan, A. (2019). In vitro antimicrobial activities of commercially available tea tree (Melaleuca alternifolia) essential oils. Current microbiology, 76(1), 108-116. 10.1007/s00284-018-1594-X

Bruno, C., \& Almeida, M. R. (2021). Óleos essenciais e vegetais: matérias-primas para fabricação de bioprodutos nas aulas de química orgânica experimental. Química Nova, 44, 899-907. https://doi.org/10.21577/0100-4042.20170722

Chin, K. B., \& Cordell, B. (2013). The effect of tea tree oil (Melaleuca alternifolia) on wound healing using a dressing model. The Journal of Alternative and Complementary Medicine, 19(12), 942-945. 10.1089/acm.2012.0787

de Groot, A. C., \& Schmidt, E. (2016). Tea tree oil: contact allergy and chemical composition. Contact dermatitis, 75(3), 129-143. 10.1111/cod.12591

Dessinioti, C., \& Katsambas, A. (2017). Propionibacterium Acnes and Antimicrobial Resistance in Acne. Clinics in dermatology, 35(2), 163-167. 10.1016/j.clindermatol.2016.10.008

Esmael, A., Hassan, M. G., Amer, M. M., Abdelrahman, S., Hamed, A. M., Abd-Raboh, H. A., \& Foda, M. F. (2020). Antimicrobial activity of certain naturalbased plant oils against the antibiotic-resistant acne bacteria. Saudi journal of biological sciences, 27(1), 448-455. 10.1016/j.sjbs.2019.11.006 
Ferreira, V. D. (2019). Toxicidade e uso de parabenos em cosméticos. https://bdm.unb.br/handle/10483/28486

Hammer, K. A. (2015). Treatment of acne with tea tree oil (melaleuca) products: a review of efficacy, tolerability and potential modes of action. International journal of antimicrobial agents, 45(2), 106-110. https://doi.org/10.1016/j.ijantimicag.2014.10.011

Hammer, K. A., Carson, C. F., \& Riley, T. V. (2012). Effects of Melaleuca alternifolia (tea tree) essential oil and the major monoterpene component terpinen4-ol on the development of single-and multistep antibiotic resistance and antimicrobial susceptibility. Antimicrobial Agents and Chemotherapy, 56(2), 909915. 10.1128/AAC.05741-11

Hoppe, A. C., \& Pais, M. C. N. (2017). Avaliação da toxicidade de Parabenos em cosméticos. Revinter, 10(3), 49-70. http://dx.doi.org/10.22280/revintervol10ed3.301

Khodaei, N., Nguyen, M. M., Mdimagh, A., Bayen, S., \& Karboune, S. (2021). Compositional diversity and antioxidant properties of essential oils: Predictive models. LWT, 138, 110684. https://doi.org/10.1016/j.lwt.2020.110684

Lam, N. S., Long, X., Su, X. Z., \& Lu, F. (2020). Melaleuca alternifolia (tea tree) oil and its monoterpene constituents in treating protozoan and helminthic infections. Biomedicine \& Pharmacotherapy, 130, 110624. https://doi.org/10.1016/j.biopha.2020.110624

Lee, C. J., Chen, L. W., Chen, L. G., Chang, T. L., Huang, C. W., Huang, M. C., \& Wang, C. C. (2013). Correlations of the components of tea tree oil with its antibacterial effects and skin irritation. journal of food and drug analysis, 21(2), 169-176. https://doi.org/10.1016/j.jfda.2013.05.007

Malhi, H. K., Tu, J., Riley, T. V., Kumarasinghe, S. P., \& Hammer, K. A. (2017). Tea tree oil gel for mild to moderate acne; a 12 week uncontrolled, open-label phase II pilot study. Australasian Journal of Dermatology, 58(3), 205-210. 10.1111/ajd.12465

Mantil, E., Daly, G., \& Avis, T. J. (2015). Effect of tea tree (Melaleuca alternifolia) oil as a natural antimicrobial agent in lipophilic formulations. Canadian journal of microbiology, 61(1), 82-88. 10.1139/cjm-2014-0667

Mazzarello, V., Donadu, M. G., Ferrari, M., Piga, G., Usai, D., Zanetti, S., \& Sotgiu, M. A. (2018). Treatment of acne with a combination of propolis, tea tree oil, and Aloe vera compared to erythromycin cream: two double-blind investigations. Clinical pharmacology: advances and applications, 10, 175. 10.2147/CPAA.S180474

Ministério da saúde. (2021). Diretrizes metodológicas: elaboração de revisão sistemática e meta-análise de ensaios clínicos randomizados. https://pesquisa.bvsalud.org/portal/resource/pt/biblio-1254554

Oliveira, A. C. M., Fontana, A., Negrini, T. C., Nogueira, M. N. M., Bedran, T. B. L., Andrade, C. R., ... \& Spolidório, D. M. P. (2011). Emprego do óleo de Melaleuca alternifolia Cheel (Myrtaceae) na odontologia: perspectivas quanto à utilização como antimicrobiano alternativo às doenças infecciosas de origem bucal. Revista brasileira de plantas medicinais, 13, 492-499. https://doi.org/10.1590/S1516-05722011000400015

Ossa-Tabares, J. C., Llanos, C. J., \& García, A. M. (2020). Evaluación de las características fisicoquímicas y de la actividad antimicrobiana del aceite del árbol de té contra Cutibacterium acnes (Propionibacterium acnes) ATCC 6919. Biomédica, 40(4), 693-701. https://doi.org/10.7705/biomedica.5122

Paviani, B. A., Trigueiro, T. H., \& Gessner, R. (2019). O uso de óleos essenciais no trabalho de parto e parto: revisão de escopo. Revista Mineira de Enfermagem, 23, 1-8. 10.5935/1415-2762.20190110

Pazyar, N., Yaghoobi, R., Bagherani, N., \& Kazerouni, A. (2013). A review of applications of tea tree oil in dermatology. International journal of dermatology, 52(7), 784-790. 10.1111/j.1365-4632.2012.05654.x

Taleb, M. H., Abdeltawab, N. F., Shamma, R. N., Abdelgayed, S. S., Mohamed, S. S., Farag, M. A., \& Ramadan, M. A. (2018). Origanum vulgare L. Essential Oil as a Potential Anti-Acne Topical Nanoemulsion-In Vitro and In Vivo Study. Molecules (Basel, Switzerland), 23(9), 2164. 10.3390/molecules23092164

Tedesco, L., Bufalo, A. C., Wietzikoski, E. C., Velasquez, P. A. G., \& Ciesca, G. M. (2014). Avaliação antibacteriana do extrato de melaleuca (Melaleuca alternifolia) frente à cepa de Staphylococcus aureus. Arquivos de Ciências da Saúde da UNIPAR, 18(2). https://doi.org/10.25110/arqsaude.v18i2.2014.5172

Williams, H. C., Dellavalle, R. P., \& Garner, S. (2012). Acne vulgaris. The Lancet, 379(9813), 361-372. https://doi.org/10.1016/S0140-6736(11)60321-8

Winkelman, W. J. (2018). Aromatherapy, botanicals, and essential oils in acne. Clinics in dermatology, 36(3), 299-305. doi:10.1016/j.clindermatol.2018.03.004

Yadav, E., Kumar, S., Mahant, S., Khatkar, S., \& Rao, R. (2017). Tea tree oil: a promising essential oil. Journal of EssEntial oil rEsEarch, 29(3), 201-213. $10.1080 / 10412905.2016 .1232665$

Zaenglein, A. L. (2018). Acne vulgaris. New England Journal of Medicine, 379(14), 1343-1352. 10.1056/NEJMcp1702493

Zhang, X., Guo, Y., Guo, L., Jiang, H., \& Ji, Q. (2018). In Vitro Evaluation of Antioxidant and Antimicrobial Activities of Melaleuca alternifolia Essential Oil. BioMed research international, 2018, 2396109. https://doi.org/10.1155/2018/2396109

Zhu, Y., Li, C., Cui, H., \& Lin, L. (2020). Encapsulation strategies to enhance the antibacterial properties of essential oils in food system. Food Control, 107856. https://doi.org/10.1016/j.foodcont.2020.107856 